\title{
El concepto de verdad en el escenario comunicativo actual: una propuesta interdisciplinar
}

The Concept of Truth in Today's Communicative Scenario: an Interdisciplinary Approach

\section{Lucía Ballesteros-Aguayo Universidad de Sevilla lballesteros@us.es}

\author{
Consuelo Aguayo Ruiz-Ruano \\ I.E.S. Alhambra (Granada) \\ consuelogran@hotmail.com
}

Resumen: El presente trabajo se plantea un acercamiento a la teoría correspondentista de la verdad con objeto de extrapolar sus conclusiones a la actividad comunicativa en un intento de impermeabilizarlas en la medida de lo posible a los efectos de la filtración de noticias falsas, los bulos o la desinformación. De esta manera se partirá del análisis filosófico que señala la necesidad de la formación y la exactitud como dos de los ingredientes que imperan en el concepto tradicional de la verdad, para llegar a la acción periodística transformada por las revoluciones operadas tanto en los soportes como en los canales de comunicación que modifican esencialmente la actividad informativa y exigen 
redefinir el propio proceso en términos de un periodismo reflexivo, interpretativo y crítico.

Palabras clave: verdad, filosofía, periodismo, formación, posverdad.

Abstract: This paper is an attempt to develop a theory of truth in order to extrapolate its conclusions to communicative activity. The aim of this is to avoid the effects of fake news, hoaxes or misinformation. In this context, a philosophical analysis has been developed to point out needs for training and accuracy. The main ingredients are to prevail the concept of truth and transform journalistic actions as a result of both media and channels of communication. Consequently, informative activity has required a redefinition process in terms of a reflexive, interpretative and critical journalism.

Keywords: truth, philosophy, journalism, training, post-truth.

Fecha de recepción: $27 / 7 / 2020$

Fecha de aceptación: 17/2/2021

Ballesteros-Aguayo, Lucía y Aguayo Ruiz-Ruano, Consuelo (2021). «El concepto de verdad en el escenario comunicativo actual: una propuesta interdisciplinan». Monograma. Revista Iberoamericana de Cultura y Pensamiento, n. ${ }^{\circ} 8$, pp. 39-58. doi: 10.36008/monograma.2021.08.1444. http: revistamonograma.com. ISSN: 2603-5839. 


\section{Introducción}

La reflexión en torno a la verdad no es exclusiva de ningún ámbito concreto ni de ninguna disciplina particular (filosofía, biología u otras), sino que constituye una constante en la racionalidad desde cualquier tipo de saber tanto teórico (su definición, su esencia, etc.) como experiencial (ligado a los hechos) o incluso axiológico (el valor de la autenticidad, de la acción moral o de los fines). Así, existen tantas dimensiones del concepto de verdad como actividades pueda llevar a cabo el ser humano.

La atribución fundamental del filósofo en su origen consistía en la indagación a propósito de la realidad con el fin de transmitir el resultado a la comunidad, por su practicidad. Entonces, la interpelación a propósito de lo que las cosas «son» (su realidad, autenticidad, substrato) quedó vinculada al ejercicio de los filósofos, hombres preparados (formados) para resolver problemas prácticos y para transmitir el resultado de sus indagaciones a la comunidad, compartirlos y publicitarlos.

La derivada teórica del acierto práctico en la resolución de los problemas (o tal vez a la inversa) condujo a una reflexión filosófica dicotómica apariencia/ realidad evidenciando que las cosas pueden ofrecer una forma de mostrarse que no es la original, la verdadera. De ahí la exigencia filosófica de indagación para descubrir (desvelar) lo oculto tras las apariencias.

Desocultar lo oculto, clarificar, esa ha sido la metáfora de la luz que jalona la historia del pensamiento: desde Parménides (la diosa «desvela») a Zambrano (la «guía» necesaria para los perple- 
jos porque «la vida no tiene por sí unidad, a lo menos no se nos hace visible, y esta es la mayor de las congojas y confusiones»), o desde Platón (el mito de la caverna) a Descartes (el criterio de certeza: «claridad y distinción»), y se convierte en la piedra angular que tradicionalmente acompaña a la verdad junto a las condiciones que posibilitan su aprehensión, es decir, su visión.

Al hilo de la experiencia de la verdad como desvelamiento aparece la necesidad de que exista un «descubridon» sólidamente formado (la diosa según Parménides, Maimónides según Zambrano, el verdadero filósofo para Platón o la Razón para Descartes), junto a una ética vocacional (Sócrates) de interlocución persuasivo-argumentativa en torno a la verdad.

Con estas mimbres (claridad, formador, formación y comunicación) afrontamos la reflexión actual en un intento de esclarecer esas ramificaciones en torno a un concepto de verdad que se muestra erosionado desde un subsuelo social comunicativo, psicológico, epistemológico como es el actual, especialmente permeable por su complejidad a errores, bulos y noticias falsas.

La importancia del alcance interdisciplinar entre periodismo y filosofía tiene su raíz en la configuración de ambas disciplinas con similares exigencias epistemológicas y deontológicas fundamentadas desde su origen en descubrir la verdad y transmitirla con veracidad, entre otras. Así se justifica que los resultados de la investigación de una de ellas sean fácilmente extrapolables a la otra promoviendo de este modo una concepción holística de la verdad.

\section{Desde el concepto corriente de verdad}

La revisión y reconstrucción del concepto de verdad desde la racionalidad actual nos plantea responder a preguntas tan inmediatas como ¿a qué tipo de verdad nos estamos refiriendo?,

Ballesteros-Aguayo, Lucía y Aguayo Ruiz-Ruano, Consuelo (2021). «El concepto de verdad en el escenario comunicativo actual: una propuesta interdisciplinan». Monograma. Revista Iberoamericana de Cultura y Pensamiento, n. ${ }^{\circ} 8$, pp. 39-58. doi: 10.36008/monograma.2021.08.1444. http: revistamonograma.com. ISSN: 2603-5839. 
¿cuáles son sus componentes?, ¿'sobre qué elementos conviene reflexionar para su reconstrucción? O ¿qué aspectos refuerzan la comunicabilidad de la verdad?

Para responder a esas cuestiones conviene delimitar previamente el tipo de verdad congruente con la actividad comunicativa del que partimos para nuestro análisis.

Muchos han sido los esfuerzos que se han llevado a cabo desde la filosofía para sinterizar las teorías de la verdad habida cuenta de su carácter poliédrico e hipercomplejo. Ello se evidencia en las numerosas clasificaciones de las teorías de la verdad, no exentas ninguna de ellas del reconocimiento de las dificultades para concluir una taxonomía con tajantes criterios de delimitación categorial.

No obstante, en nuestro caso hay dos motivos que nos inducen a situar este trabajo a partir de las llamadas teorías correspondentistas de la verdad: que son las teorías más generalizadas y de mayor transcendencia histórica, y que la práctica periodística (en especial en un mundo de verdades alternativas y noticias falsas) es congruente con el debate en torno a la veracidad de los hechos.

El carácter referencial de la teoría correspondentista ya lo señalan Frápolli Sanz y Nicolás Marín (1997b: 16) cuando afirman que las teorías de la correspondencia:

Son sin duda las que mayor fuerza y vigencia histórica han tenido. Tan es así, que la concepción prototípica de la correspondencia se ha convertido en la referencia respecto a la cual se definen otras concepciones alternativas de la verdad, sean pragmáticas, coherentistas o hermenéuticas.

Una vez justificado el punto de partida, explicitaremos cada uno de los cuatro apartados que a juicio de Frápolli Sanz y Ni- 
colás Marín debe recoger toda teoría de la verdad (definición, criterio, semántica y pragmática), y los aplicaremos a la función periodística a fin de conseguir nuestro objetivo de pautar actuaciones que conduzcan a fortalecer la verdad.

Por un lado, en relación con la definición de la verdad como correspondencia diremos que es ampliamente conocida y generalmente admitida de manera intuitiva (y a buen seguro resulta comprensible para la mayoría de personas). Una de las formulaciones más investigadas de esta teoría en la actualidad se debe a Tarski en Frápolli Sanz y Nicolás Marín (1997b: 69) para quien «la verdad de una oración consiste en su acuerdo (o correspondencia) con la realidad».

Por otro lado, si consideramos el criterio de certeza, lo más destacable para este trabajo es señalar que cualquier conflicto en torno a la verdad puede resolverse confrontándolo con la realidad de tal modo que, si el enunciado satisface la realidad, es verdadero; y en caso de que no la satisfaga es falso (todo ello al margen de la clasificación de subcategorías — semánticas y no semánticas - dentro de la teoría correspondentista de la verdad). Parafraseando a Aristóteles «decir de lo verdadero que es verdadero y de lo falso que es falso».

Dicha forma tan intuitiva de aplicar el criterio resulta factible para los ciudadanos de a pie pues no plantea de antemano ningún problema para diferenciar lo verdadero de la falso; de hecho, preguntar por la verdad en la mayoría de las ocasiones es preguntar por la verdad como correspondencia. Así nos lo indica Tarski en la síntesis que recogen Frápolli Sanz y Nicolás Marín (1997b: 94):

...si le preguntáramos a un muchacho de la escuela secundaria, o a un adulto inteligente sin preparación filosófica especial, si

Ballesteros-Aguayo, Lucía y Aguayo Ruiz-Ruano, Consuelo (2021). «El concepto de verdad en el escenario comunicativo actual: una propuesta interdisciplinar». Monograma. Revista Iberoamericana de Cultura y Pensamiento, n. ${ }^{\circ}$ 8, pp. 39-58. doi: 10.36008/monograma.2021.08.1444. http: revistamonograma.com. ISSN: 2603-5839. 
considera que una oración es verdadera si concuerda con la realidad, o si designa una situación existente, puede resultar simplemente que no comprenda la pregunta; por consiguiente su respuesta, cualquiera que sea, carecerá de valor para nosotros. Pero su respuesta a la pregunta acerca de si admitiría que la oración «está nevando» pueda ser verdadera aun cuando no esté nevando, o falsa aunque esté nevando, sería, naturalmente, muy importante para nuestro problema.

Por esto, nada me sorprendió (en una discusión dedicada a estos problemas) enterarme de que en un grupo de personas preguntadas solo el 15 por 100 concordó en que «verdadero» significa para ellos «concordante» con la realidad, en tanto que el 90 por 100 convino en que una oración tal como «está nevando» es verdadera si, y solo sí, está nevando.

Conviene destacar que la noción correspondentista de la verdad se halla ligada a una situación determinada, es puntual («está nevando»), esto es, la verdad o falsedad del enunciado puede concordar o confirmarse en un momento dado concreto y de forma presencial, el resto pertenece al relato.

Pero este criterio tan intuitivo y fácilmente aplicable desde la pragmática ciudadana no está exento de problemas desde una perspectiva semántica y metalingüística, a partir de la cual la problemática es más amplia.

Aun a riesgo de simplificar la compleja reflexión que relaciona la adecuación entre verdad y lenguaje, el presente trabajo pone de relieve tres problemas por su interés para la comunicación periodística:

1. En primer lugar, es preciso advertir que nos estamos refiriendo a la verdad de una oración pronunciada por un hablante en la que intervienen factores como su riqueza léxica, el dominio o no del campo semántico, el conocimiento 
del código específico, etc. Esta lingüisticidad del criterio conviene considerarla en los procesos comunicativos especialmente y ante todo porque acrecienta o disminuye la posibilidad de error, y se deriva en gran medida de la formación.

2. También debemos considerar la presencia de elementos extralingüísticos en la comprensión del mensaje por parte del receptor como son: las habilidades individuales gestuales o corporales, el tono de la voz, su imagen corporal, el dominio de los silencios, y en general todo lo relacionado con la retórica, junto a otros elementos del contexto. Algunos de esos elementos son insalvables, otros pueden ser aprendidos a través de la formación.

3. En tercer lugar cabe destacar que esa adecuación se puede resolver con relativa facilidad cuando nos referimos a una función meramente descriptiva del lenguaje, pero se vuelve problemática si tenemos en cuenta funciones del lenguaje como la emotiva, conativa o poética, contextos en los que es latente la posibilidad de permear fake news, bulos o discursos posverdaderos en los procesos comunicativos, en especial aquellos que se ligan —estén relacionados o no- con las emociones.

Concluimos por fin que gran parte de la aplicación de la pragmática correspondentista pertenece al relato, de ahí la importancia de la formación del relator en su función informativa, quien para ofrecer información de calidad requiere: dominar el campo semántico, verificar las fuentes, contrastar la información y elegir el medio más idóneo para su difusión, entre otras cuestiones.

Testificar la veracidad de la noticia, contar «lo visto y oído», desplazarse al lugar del hecho noticioso para atestiguar ocular,

Ballesteros-Aguayo, Lucía y Aguayo Ruiz-Ruano, Consuelo (2021). «El concepto de verdad en el escenario comunicativo actual: una propuesta interdisciplinar». Monograma. Revista Iberoamericana de Cultura y Pensamiento, n. ${ }^{\circ} 8$, pp. 39-58. doi: 10.36008/monograma.2021.08.1444. http: revistamonograma.com. ISSN: 2603-5839. 
visual o auditivamente con dispositivos idóneos no siempre es posible (y menos aún en un mundo global), por lo que cobra más importancia la exigencia ética de formación y contrastabilidad. Es por ello por lo que se impone incidir con firmeza en la reflexión de las garantías del relato que contribuya a la reducción del error y la disolución de noticias falsas, a saber: la exactitud que garantiza la veracidad, y la formación del especialista que promueve la calidad informativa.

\section{Exigencias en torno a la verdad como correspondencia: la exactitud y la formación}

Una vez resueltas algunas de las preguntas formuladas al inicio en torno al tipo de verdad a considerar, su criterio y sus componentes pragmáticos conviene señalar dos elementos a tener en cuenta para un periodismo de calidad: la formación y la exactitud. Ahora bien, la pragmática en torno a la verdad evidencia una diferencia entre el ámbito de lo privado, en el que hay más exigencias de veracidad informativa («exijo saber exactamente cuál el verdadero estado de mi enfermedad»), y el ámbito de lo público, en el que las exigencias de veracidad se atenúan, se desvanecen o incluso en ocasiones desaparecen.

Ello demuestra la complejidad del proceso informativo en el que intervienen además de la formación periodística otros muchos aspectos a considerar como pueden ser: la motivación, el interés o la formación de la ciudadanía en el cribado de información de calidad.

Por consiguiente, aunque no es el único factor que inhibe la penetración de informaciones falsas, la formación de la ciudadanía en los medios y para los medios permite: conocer los soportes para seleccionar el más idóneo valorando sus principios, con- 
trastar la noticia en distintos soportes para garantizar su veracidad, descartar las fuentes informativas dudosas, y comprender los fines de la información para valorar el alcance de la noticia, entre otros aspectos.

El interés formativo de los consumidores de noticias hoy en día surge tímidamente incluso en el seno de la propia industria comunicativa. Es el caso, por ejemplo, de las iniciativas de verificación o el hecho de que la mayoría de los medios incorpora una sección especializada en detectar bulos y en verificar y/o contrastar noticias falsas (Magallón-Rosa, 2018) en aras de apostar por la calidad informativa y de contribuir con la labor de servicio público inherente al periodismo tradicional. Más aún, a raíz de la proliferación de fake news y de la problemática de la desinformación, esta función social y pública de verificación y contrastación se ha convertido en un rasgo distintivo del periodismo profesional frente a otras prácticas como pueden ser el «pseudoperiodismo», entendido como la imitación de formatos periodísticos como medio de transmisión de noticias falsas o inventadas (Burgueño Muñoz, 2008); el «periodismo de filtraciones, sensacionalismo, intereses, declaraciones acusatorias y cámara oculta» en contraposición al periodismo de investigación (Marta-Lazo y Fernández Montañés, 2016); o el «periodismo amateur digital», identificado con la profusión de noticias «improvisadas» que resultan «más atractivas» — con frecuencia por su componente emotivo- para el público en general.

La información de muchos enclaves de Internet resultó falsa o distorsionada, producto de noticias improvisadas carentes de contraste o del necesario contexto, por un indisimulado sesgo ideológico. Esta información cumplió la función emocional clásicamente atribuida a los rumores en situaciones de crisis y carencia de clarificación oficial [...] La escasa fiabilidad de bue- 
na parte de este «periodismo amateur digital» plantea el problema de la «competencia desleal» sufrida por el periodismo profesional cuando esas otras «noticias improvisadas» resultan más atractivas y «creíbles» para una población en estado de shock tras un desastre. (Dader García, 2006: 44)

Derivar la comprensión de la verdad hacia distintas propuestas formativas vocacionales no es nueva, al contrario, en la historia de la filosofía abundan las propuestas didácticas con mayor o menor fortuna en orden a potenciar la formación de la ciudadanía en la verdad, a pesar de la resistibilidad del ser humano a aceptar propuestas pedagógicas que requieran cambios significativos en las convicciones arraigadas, en las opiniones personales (fruto de un egoísmo exacerbado: «yo soy el depositario de la verdad», cualquier otra opinión ni es discutible ni es aceptable y de una formación acrítica) o en la cosmovisión derivada.

\subsection{El mito de la caverna como propuesta pedagógica} para el descubrimiento de la verdad

Una de las propuestas pedagógicas más interesantes por su incidencia en la formación (instrucción) es la que describe Platón en el Mito de la caverna, y que recoge más tarde y reinterpreta Heidegger desde una revisión filológica de la terminología platónica. Siendo conscientes de la simplificación que supone un análisis tan complejo que obligaría a extendernos más allá de estas líneas, ofrecemos una sucinta exposición de la problemática planteada, particularmente por las consecuencias que se derivan de su descripción de la alegoría de la caverna a fin de valorar la formación y la exactitud como ingredientes prototípicos diacrónicos de la verdad. 
La preocupación de Heidegger por la verdad queda patente en la extensa y prolífica producción que incluye además de la exégesis desarrollada en El ser y el tiempo (1951) varios artículos en los que expone su esencia, el criterio y su exégesis como Conferencias y articulos (1994) o De l'essence de la vérité (1948) o Questions I et II (1968).

Analizamos el concepto corriente de la verdad desde la hermenéutica histórica de Heidegger en su recorrido por la tradición filosófica occidental según las líneas expuestas en dos obras fundamentales: por un lado, Questions I et II (1968), y, por otro, la síntesis que ofrecen Frápolli Sanz y Nicolás Marín en Teorías de la verdad en el siglo XX (1997b).

En primer lugar, Heidegger destina un parágrafo al análisis histórico de la verdad correspondentista titulado «El concepto corriente de verdad» en el que establece como punto de partida la adecuación como la posición fundamental que recorre la concepción tradicional de la verdad para la cual «lo verdadero es lo real». Esta es la raíz primaria de la verdad, y apunta a identificarla con el resultado del desvelamiento.

Según Heidegger (1968) la gradación que establece Platón en la alegoría de la caverna en cada una de las estancias de los prisioneros es proporcional a los grados de formación que deben de ir adquiriendo para la aprehensión de la verdad.

Así, la narración de Platón resulta singular en relación con «lo real», pues en el primer estadio se toma como real lo que rodea a los prisioneros (lo real aún está tapado, velado) y, aunque esto no sea más que un mundo de sombras, es lo más cercano que tienen los prisioneros ya que las ideas que constituyen «el ser de las cosas» en ese momento se hallan muy lejanas.

En el segundo estadio, lo que se entiende por real va cambiando en relación proporcional al ascenso a otra estancia pero 
lo importante no es la relativa apertura (desvelamiento) del ser, sino que el prisionero debe adaptarse a mirar hacia otro tipo de realidad y sufrir la angustia derivada de tener que acostumbrarse a ese nuevo real.

Adaptarse a lo real solo se consigue con una buena formación. Esto es, no porque la verdad esté al descubierto el prisionero es capaz de captarla, por tanto, no es suficiente con el desvelamiento, resulta imprescindible además la formación ( $\pi \alpha \iota \delta \varepsilon i \alpha)$ para poder conquistar la verdad (1968: 134): «Cette nouvelle orientation, cette adaptation de l'être de l'homme au domaine qui lui est chaque fois assigné, constitue l'essence de ce que Platon appelle la $\pi \alpha|\delta \varepsilon i \alpha\rangle$.

En el tercer estadio se encuentra el máximo desvelamiento tras la liberación de las cadenas y la visión de lo verdaderamente ente, pero la verdadera liberación no proviene del desencadenamiento, advierte Platón, sino que es resultado de la formación ( $\pi \alpha i \delta \varepsilon i \alpha)$ que capacita la adaptación de la mirada a la «claridad» que irradia el ser real. Y así se entiende que lo más importante es la justeza, la exactitud de la mirada que se adapta a la luz que irradia el máximo ente, el Bien. En palabras de Heidegger (1968: 135): «La force symbolique du "mythe de la caverne" est centrée sur le dessein de rendre l'essence de la $\pi \alpha \iota \delta \varepsilon i \alpha$ visible et connaissable à travers les formes sensibles d'une histoire racontée».

Lo que posibilita la aprehensión de la verdad en definitiva no es la visión de lo real, sino la formación. Habida cuenta de que el descenso del prisionero a la caverna con fines educativos se cuestiona problemático —esto es, «ayudar» a ver a los demás prisioneros no es tan fácil—, el mediador precisa de buenas dotes para comunicar, además de realizar un gran esfuerzo para persuadir en la verdad, toda vez que la realidad está lejana. 
En síntesis, el mito platónico nos permite concluir que el aprendizaje de la verdad es fruto de las destrezas comunicativas de un verdadero profesional bien formado y de la apertura de la ciudadanía a la verdad.

\subsection{La verdad concebida como autenticidad}

A partir de esta inflexión platónica la tradición filosófica señala en la adecuación un camino de ida y otro de vuelta, de manera que las cosas se pueden adecuar al entendimiento, o bien el entendimiento debe adecuarse a las cosas para conocerlas. Ello abre numerosas posibilidades a la adecuación.

Estas posibilidades nos aproximan a otra raíz de la verdad tradicional concebida como autenticidad a la que también Heidegger alude en su análisis en De la esencia de la verdad (Nicolás Marín y Frápolli Sanz, 1997b) cuando señala como ejemplo de la verdad correspondentista la concordancia con lo que «le corresponde», y así asevera que para el concepto corriente de verdad

lo verdadero es lo real. De acuerdo con esto hablamos de oro verdadero a diferencia del falso. El oro falso no es realmente lo que parece. Es solo una «apariencia» y por tanto irreal (unwirklicb). Lo irreal es tenido como lo contrario de lo real. Pero el oro aparente es también algo real. (1997b: 401-402)

De esta concepción cabe destacar para el presente trabajo la importancia que el concepto de verdad otorga a la formación como esencia de la garantía de veracidad pues solo el orfebre especializado, el profesional formado, podrá determinar con exactitud la justeza de una pieza para validar su autenticidad conociendo el modelo. Por consiguiente, su testimonio es imprescindible para no errar.

Ballesteros-Aguayo, Lucía y Aguayo Ruiz-Ruano, Consuelo (2021). «El concepto de verdad en el escenario comunicativo actual: una propuesta interdisciplinan». Monograma. Revista Iberoamericana de Cultura y Pensamiento, n. ${ }^{\circ} 8$, pp. 39-58. doi: 10.36008/monograma.2021.08.1444. http: revistamonograma.com. ISSN: 2603-5839. 
Testimoniar es emitir un juicio («esto es oro verdadero» o «esto es oro falso»), luego, en definitiva, es el especialista bien formado quien decide y dictamina mediante su veredicto, su juicio se corresponde con la verdad.

Estas nuevas posibilidades emergen de la reflexión a propósito de la pronunciación de la verdad en torno a la «realidad». De hecho cuando se afirma que «porque tú eres blanco, nosotros, los que lo afirmamos, nos ajustamos a la verdad» se sugiere que la adecuación, la justeza es anterior y viene impuesta por la experiencia.

Esta «anticipación» de la verdad a la que nuestro enunciado debe ajustarse también la encontramos en la reflexión que desde otra perspectiva hace Zubiri (1978: 13) de la verdad como adecuación: «Dicho de otro modo, la verdad es, según la fórmula tradicional, un acuerdo del pensamiento con las cosas. Todo el problema de la ciencia estriba, pues, en llegar a un acuerdo cada vez mayor con la mayor cantidad de cosas».

Zubiri señala, además, las condiciones que deben cumplirse para acordar la verdad: que las cosas «han de estar presentes al hombre»; que la pregunta por la verdad se lleva a cabo desde una dimensión de la realidad, es decir, «presupone un sistema de cuestiones previas con que la inteligencia afronta la realidad»; y que la pregunta «nace de la estructura total de la situación de la inteligencia humana». Pero todo ello presupone que el horizonte desde el que se plantea el acuerdo es inexorable, es decir, previo a pensar en la verdad de las cosas se está inmerso en ellas inexorablemente.

El pensamiento, pues, es cierto que tiene que conquistar cosas, pero es porque está ya previamente moviéndose en ellas. Y aquí está el grave equívoco a que antes aludía. La verdad, como un acuerdo con las cosas, supone siempre un previo estar

Ballesteros-Aguayo, Lucía y Aguayo Ruiz-Ruano, Consuelo (2021). «El concepto de verdad en el escenario comunicativo actual: una propuesta interdisciplinan». Monograma. Revista Iberoamericana de Cultura y Pensamiento, n. ${ }^{\circ}$ 8, pp. 39-58. doi: 10.36008/monograma.2021.08.1444. http: revistamonograma.com. ISSN: 2603-5839. 
en ellas. Hay una verdad (y si se quiere también una falsedad, dejemos el problema) radical y primaria de la inteligencia: su constitutiva inmersión en las cosas. (Zubiri, 1978: 21)

La verdad real que defiende Zubiri está en consonancia con las características de la realidad que son a su juicio: totalidad, coherencia, y estabilidad, y ellas se ratifican en la intelección en «manifestación», «firmeza»y «constatación».

En suma, lo que Zubiri plantea es que la exactitud, la justeza, es relativa a las cosas, los conceptos humanos son los que deben ajustarse a ellas para conquistar su verdad y publicarla: esa es precisamente la labor del especialista, del científico. A ello añade que la diferencia entre «conformidad» y «adecuación» nos conduce a una nueva concepción de la verdad que la filosofía tradicional no había deparado: la verdad como «cumplimiento».

En conclusión: las tres raíces históricas de la verdad (como desvelamiento, como autenticidad y como adecuación) apuntan a la necesidad de especialistas bien formados que garanticen la correspondencia, la credibilidad y la veracidad de la calidad informativa.

Esto es especialmente relevante en el contexto comunicativo actual en el que la influencia de la formación en la calidad informativa se torna en una moneda de dos caras de distinta gradación y exigencia ética de manera que la práctica periodística imbrica tanto a los profesionales de la información como a los consumidores, máxime cuando habitualmente se intercambian los roles entre consumidores (prosumidores) y productores.

Educar en los medios es uno de los elementos clave para: distinguir en el hecho noticioso la verdad del simulacro (bulos, noticias falsas, etc.), contrastar las noticias en orden a seleccionar las que muestren credibilidad y fidelidad, discriminar formativa- 
mente las fuentes dudosas de las que no lo son, y discernir entre todas las noticias que se presentan cuáles son auténticas y cuáles son falsas para difundirlas o desecharlas.

\section{Conclusiones}

Este trabajo muestra la fertilidad de los resultados de la reflexión interdisciplinar entre filosofía y comunicación en torno a los supuestos de la verdad tradicional: su definición, su criterio y la pragmática resultante, con implicaciones en el proceso comunicativo.

También se evidencia que la verdad correspondentista tradicional es susceptible de implementar las exigencias veritativas de la comunicación periodística al encontrarse esta ligada a la veracidad de los hechos y a la contrastación de las fuentes.

Se establecen además dos características significativas de la verdad correspondentista: la necesidad de la formación, y la exigencia de exactitud (ambas ligadas también a la verdad como adecuación y a la verdad como autenticidad) en cuya virtud se reivindica argumentativamente la necesidad de los especialistas que atestigüen la autenticidad y veracidad del hecho noticioso.

Se explicita a la vez tres problemas derivados del carácter discursivo de la verdad como correspondencia que inciden de manera especial en el ejercicio profesional del periodismo: su lingüisticidad, su contextualidad y la profusión de noticias falsas.

Concluimos que la robustez de un modelo educativo basado en la formación para la verdad promueve la inhibición de bulos, fake news o noticias falsas en la medida en que abarca —al menos - los dos vértices del proceso comunicativo, a saber: los productores de la información y los consumidores. 
De igual modo, la contrastación, concordancia y autenticidad que promueve la educación para la verdad reorienta la apertura de los consumidores de la información hacia una visión más crítica y reflexiva en un mundo plural.

Finalmente se concluye la necesidad de reorientar la acción formativa de los profesionales de la comunicación hacia una educación para los medios en la sociedad en su conjunto habida cuenta de la influencia que ejerce la comunicación en la edificación de cosmovisiones compartidas.

Ballesteros-Aguayo, Lucía y Aguayo Ruiz-Ruano, Consuelo (2021). «El concepto de verdad en el escenario comunicativo actual: una propuesta interdisciplinan». Monograma. Revista Iberoamericana de Cultura y Pensamiento, n. ${ }^{\circ} 8$, pp. 39-58. doi: 10.36008/monograma.2021.08.1444. http: revistamonograma.com. ISSN: 2603-5839. 


\section{REFERENCIAS BIBLIOGRÁFICAS}

Burgueño Muñoz, J. M. (2008). La invención en el periodismo informativo. Barcelona: UOC.

Castells, M. (2006). La sociedad red: una visión global. Madrid: Alianza Editorial.

Conford, F. M. (1983). La teoría platónica del conocimiento. Barcelona: Paidós.

Dader García, J. L. (2006). «Cibercomunicación y pseudoperiodismo en el 11-M: patologías de crisis en una democracia débil». Comunicación y pluralismo, pp. 35-60. Disponible en: https:/ / summa.upsa.es/pdf.vm?id=0000029111\&page $=1 \&$ search $=\&$ lang $=$ es [Consulta: 2 febrero 2021].

De Pablos Coello, J. M. (2008). «El frenesí comunicativo como desinformación». Comunicar, 16(31), pp. 173-179. Disponible en: https://recyt.fecyt.es/index.php/comunicar/article/ view/26092 [Consulta: 2 febrero 2021]. doi: https://doi. org/10.3916/c31-2008-01-022.

Heidegger, M. (1948). De l'essence de la vérité. Trad. de A. de Waehlens y W. Biemel. París: E. Nauwelaerts / Lovaina: J. Vrin.

— (1951). El ser y el tiempo. Trad. de José Gaos (1. a ed. en español). México: Fondo de Cultura Económica.

- (1968). Questions I et II. Trad. de K. Axelos et al. París: Gallimard.

- (1994). Conferencias y artículos. Barcelona: Ediciones Del Serbal.

Magallón-Rosa, R. (2018). «Nuevos formatos de verificación. El caso de Maldito Bulo en Twitter». Sphera Publica, 1(18), pp. 41-62. Disponible en: http://sphera.ucam.edu/index. php/sphera-01/article/view/341 [Consulta: 2 febrero 2021]. 
Marta-Lazo, C. y Fernández Montañés, M. E. (2016). «El pseudoperiodismo de investigación en las cadenas generalistas de televisión en España». Ecos de la Academia, 3, pp. 75-89. Disponibleen:https://core.ac.uk/download/pdf/289985881. pdf [Consulta: 2 febrero 2021].

Morin, E. (1967). El espiritu del tiempo. Madrid: Taurus.

Nicolás Marín, J. A. (2019). «Posverdad: cartografía de un fenómeno complejo». Diálogo Filosófico, 105, pp. 302-340.

Nicolás Marín, J. A. y Frápolli Sanz, M. J. (1997a). «Teorías actuales de la verdad». Diálogo Filosófico, 38, pp. 148-178.

— (1997b). Teorías de la verdad en el siglo XX. Madrid: Tecnos.

VAттімо, G. (1986). Introducción a Heidegger. Barcelona: Gedisa.

Zambrano, M. (1993). Hacia un saber sobre el alma. Madrid: Alianza Editorial.

Zubiri, X. (1978). Naturaleza, historia, Dios. (7. ed.). Madrid: Editora Nacional.

Ballesteros-Aguayo, Lucía y Aguayo Ruiz-Ruano, Consuelo (2021). «El concepto de verdad en el escenario comunicativo actual: una propuesta interdisciplinar». Monograma. Revista Iberoamericana de Cultura y Pensamiento, n. ${ }^{\circ} 8$, pp. 39-58. doi: 10.36008/monograma.2021.08.1444. http: revistamonograma.com. ISSN: 2603-5839. 